Version of November 6, 2019

\title{
Micron-sized double emulsions and nematic shells generated via tip streaming
}

\author{
Kunyun He, ${ }^{1}$ Francisco Campo-Cortés, ${ }^{2}$ Martyna Goral, ${ }^{1}$ Teresa López-León, ${ }^{1}$ and José Manuel Gordillo 2 , \\ ${ }^{1}$ EC2M, UMR 7083 Gulliver, CNRS, ESPCI Paris, \\ PSL Research University, 10 Rue Vauquelin, 75005 Paris, France. \\ ${ }^{2}$ Área de Mecánica de Fluidos, Departamento de Ingenería Aeroespacial y Mecánica de Fluidos, \\ Universidad de Sevilla, Avenida de los Descubrimientos s/n 41092, Sevilla, Spain.
}

(Dated: November 6, 2019)

Here we show that the so called Confined Selective Withdrawal technique 1 can be used to produce compound drops whose radii and thicknesses can be easily decreased down to length scales of the order of $5 \mu \mathrm{m}$, and even smaller. This method is based on the generation of a highly stretched thin coaxial jet of two immiscible fluids that flow inside a third bulk co-flowing fluid under creeping flow conditions. The subsequent capillary breakup of such coaxial jet produces, in a single step, compound droplets at rates that can be easily modulated to values of the order of $10 \mathrm{KHz}$. Using algebraic equations deduced using slender body theory, we report that the production frequency, the diameter and the shell thickness follow well defined scaling laws, enabling us to generate, from milimeter-sized injection tubes, and in a highly controlled way, uniformly-sized double emulsions with the smallest diameters reported up to date. We illustrate our results by producing micron-sized nematic shells with radii below 5 microns and show that the radius of curvature of the surface and the shell thickness can be fixed independently. 


\section{INTRODUCTION}

Double emulsions are currently used in fields as diverse as pharmacology, food science or cosmetics with the purpose of encapsulating different types of active ingredients [2 5]. They also find applications in material science, where they can be used as building blocks of new materials [6]. However, despite their clear relevance in many different technologies, the controlled generation of compound drops with a diameter smaller than a few tens of microns still remains a technological challenge [2, 3, 9].

Here we explore the possibility of producing compound droplets with diameters below $10 \mu \mathrm{m}$ through the capillary breakup of the highly stretched compound jet which is issued from the tip of a conical drop when an outer coflowing stream surrounds the meniscus [1, 10, 11. The configuration employed here, referred to as Confined Selective Withdrawal (CSW) in Evangelio et al [1, 12, involves the coaxial co-flow of two immiscible fluids surrounded by a third outer co-flowing fluid, which imposes a strain flow and also a favourable pressure gradient over the resulting compound jet. Under appropriate conditions, a thin jet is ejected from the tip of a conical-shaped meniscus formed at the end of a millimetric injection needle, a situation which resembles that observed in the electrospraying of charged liquids [2, 3, 13]. The micro-jet emerging from the cone eventually breaks into double emulsions due to the growth of capillary instabilities.

In [1] it is shown that the jet diameter, and thus the diameters of the drops composing the double emulsion, is not set by geometrical dimensions, but mostly by the pressure gradient and the flow rates, which in principle could be tuned at will, offering great potential for producing micron-sized droplets. While previous works have focused on establishing the scaling laws governing the double-emulsion generation process [1], here we focus on establishing the limits of the production regimes. We show that this method allows for the production of double emulsions in a large range of sizes at high frequency. In particular, we show here for the first time that it is possible to produce, using injection capillaries with diameters of the order of $1 \mathrm{~mm}$, in a single step and in a highly controlled way, compound droplets with outer diameters below $10 \mu \mathrm{m}$ and thicknesses of $1 \mu \mathrm{m}$, an unprecedented result which is unachievable by other similar methods that make use of either glass capillary devices [4, 14] or of PDMS-based microfluid chips [5], as it will be discussed in the following sections.

Here, we will illustrate the capability of the CSW technique to produce compound drops with diameters below 10 microns using, as intermediate fluid, a nematic liquid crystal, a choice motivated by the applications related to material science. Indeed, it has been suggested [15 that it might be possible to ascribe valence and specific directional bonding to standard spherical colloidal particles by coating their surfaces with a nematic shell. When nematic order is forced to develop on a curved surface, geometrical frustrations lead to the formation of topological defects, which appear symmetrically positioned on the particle surface [16. These topological defects are not only mathematic concepts, but also physical entities with properties intrinsically different from the nematic phase in which they appear, which can be functionalized with ligands to eventually induce bonds between particles [17]. Such functionalized defects would somehow act as atomic orbitals: the number of defects would determine the valence of the particle and the defect arrangement would determine the bonding directionality. The prospect of producing mesoscopic "atoms" by confining a liquid crystal to a spherical shell has triggered an important amount of research in the last years [6 8, 18.

The experiments reported here illustrate the capability of the CSW technique to produce the smallest compound droplets produced by hydrodynamics means reported so far. Notice that the results reported are not restricted to the particular case of liquid crystals: it is equally valid to encapsulate a drop of a newtonian fluid within a drop of another newtonian fluid, an assertion supported by the fact that the experimental results obtained will be rationalized using an extension of the classical slender-body theory developed decades ago for the case of newtonian fluids (see, e.g. [19] and references therein).

\section{EXPERIMENTAL SET-UP}

The set-up, sketched in Fig,1] consists of a coaxial needle through which two immiscible fluids, inner and middle, are injected into a pressurized chamber at respective flow rates $Q_{i}$ and $Q_{m}$. The injector is aligned with the axis of a square extraction tube, of width $L$, and both injection and extraction tubes are separated by a distance $\sim O(L)$. A flow rate $Q_{o}$ of an outer liquid with density $\rho_{o}$ and viscosity $\mu_{o}$, which constitutes the continuous phase, and immiscible with the intermediate fluid, flows into the chamber, where the pressure $P_{o}$ is larger than atmospheric, $P_{a}$. The imposed pressure difference $P_{o}-P_{a}$ fixes the mean velocity $U$ at the square extraction tube, and also generates a pressure gradient from the exit of the injection tube towards the entrance of the extraction duct. The flow of the outer liquid, from the chamber to the atmosphere through the extraction tube, produces the suction of the inner and 
middle fluids, favoring the formation of a compound capillary jet which strongly narrows downstream. The outer flow

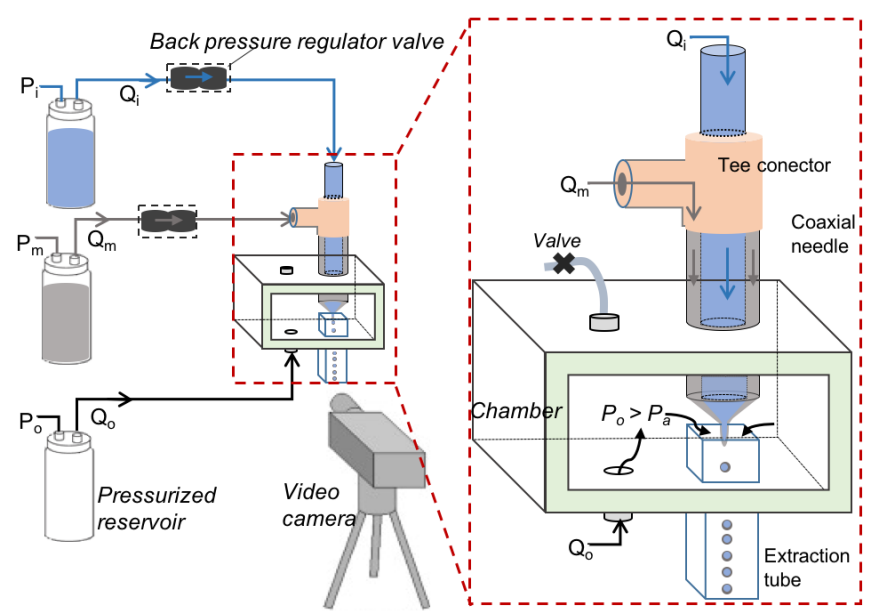

FIG. 1: Sketch of the experimental setup for the production of nematic liquid crystal shells. The coaxial injector is composed of an inner capillary tube with an inner diameter of $d_{i}=0.4 \mathrm{~mm}$ and length of $12.5 \mathrm{~cm}$. The middle fluid flows through a second tube, coaxial with the innermost one, of diameter $d_{o}=1.1 \mathrm{~mm}$ and length $5 \mathrm{~cm}$. The square glass capillary tube possesses a length $L_{T} \simeq 4 \times 10^{-2} \mathrm{~m}$ and a width $L=10^{-3} \mathrm{~m}$. A square section extraction tube

is employed to avoid optical distorsions. The coaxial alignment between the injection and extraction tubes is ensured by checking the front and side views of the setup provided by two cameras placed perpendicularly to each other. The pressure chamber is designed to resist pressure differences $P_{0}-P_{a}$ in the range of 0-5 bars (0-500 KPa). The sketch shows one of the two possible set-ups exlored, in which fluids are injected by imposing manually different gas pressures over the three immiscible liquids stored in different reservoirs.

rate, $Q_{o}$, is calculated measuring the volume of liquid exiting the pressurized chamber in a given interval of time. A precise control of this quantity is achieved using a high precision air pressure regulators (Festo) to fix the pressure difference $P_{o}-P_{a}$ between the pressurized chamber and the atmosphere; all pressure differences are measured using digital manometers (Digitron). Since the Reynolds number characterizing the flow in the extraction tube verifies the condition $R e_{o}=\rho_{o} U L / \mu_{o} \lesssim 1$, the outer flow rate $Q_{o}=U L^{2}$ is expected to depend linearly on $P_{o}-P_{a}$. Indeed, Poiseuille's equation relating the flow rate with the pressure drop along a pipe, yields:

$$
\begin{aligned}
& U L^{2} \simeq \frac{\pi L^{4}}{128 \mu_{o}} \frac{P_{o}-P_{a}}{L_{T}} \rightarrow U \simeq K \frac{L}{\mu_{o}}\left(P_{o}-P_{a}\right) \\
& \text { with } \quad K \simeq \frac{\pi}{128} \frac{L}{L_{T}}
\end{aligned}
$$

and where the square tube has been approximated by a cylinder of diameter $L$ and length $L_{T}$. To deduce Eq, 1 we have also taken into consideration that the flow rate $Q_{o}$ is such that $Q_{o} /\left(Q_{i}+Q_{m}\right) \gg 1$, being this the reason why Eq.1 is independent of both $Q_{i}$ and $Q_{m}$. Eq.1 is verified experimentally in Fig. 2 and thus, from now on, the expected scaling laws for the shell production frequency and diameter will be expressed as a function of the mean velocity $U$, instead of $P_{o}-P_{a}$. Owing to the fact that the hydrostatic pressure difference is much smaller than the pressure drop at the entrance of the extraction tube namely, $\Delta \rho g \mathrm{E}^{2} /\left(32 \mu_{o}\right) \ll 1$ with $\Delta \rho$ the characteristic density jump between outer and inner fluids, the experimental results will not be affected, in a first approximation, by buoyancy effects.

For most of the experiments reported here, the flow rates $Q_{i}$ and $Q_{m}$ are controlled using high precision syringe pumps (Harvard Apparatus). However, part of the experimental data have been collected using pressure regulators (Festo) to impose manually the pressure differences $P_{i}-P_{o}$ and $P_{m}-P_{o}$. To prevent fluctuations in both $Q_{i}$ and $Q_{m}$ when using pressure regulators, our experimental set-up incorporates long and thin copper tubes of $100 \mu \mathrm{m}$ inner diameter to impose a large pressure drop along the feeding lines. Either a back pressure regulator valve or a switch on-off valve are used to limit the flow to one direction, preventing the meniscus from retracting when pressure changes are introduced in the system, see Fig.1. Images and videos are captured using a Phantom V711 high-speed camera which, depending on the value of $U$, operates between 4000 and 10000 frames per second (fps). The double emulsions produced are collected by placing a glass slide underneath the extraction tube. 


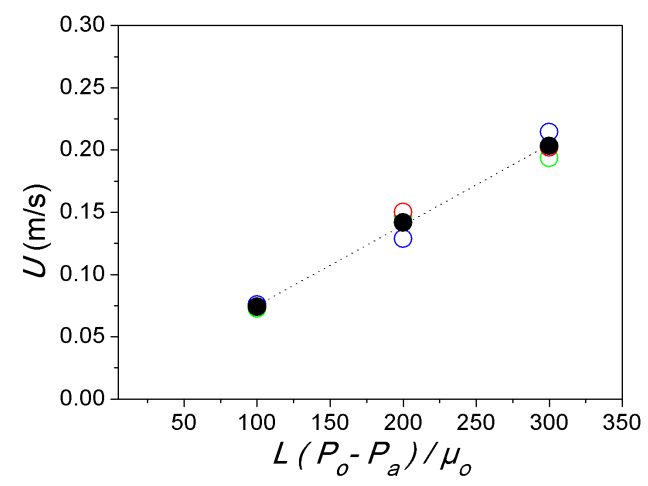

FIG. 2: The liquid velocity at the extraction tube, $U$, depends linearly on the pressure difference $P_{o}-P_{a}$, as expected from the fact that $R e_{o}<1$ (see Eq. 1). The blue, red, and green circles represent, respectively a fixed $Q_{i}$ at $0.1 \mathrm{ml} / \mathrm{h}, 0.5 \mathrm{ml} / \mathrm{h}$, and $1 \mathrm{ml} / \mathrm{h}$, while the black dots represent the prediction given in Eq. 1 .

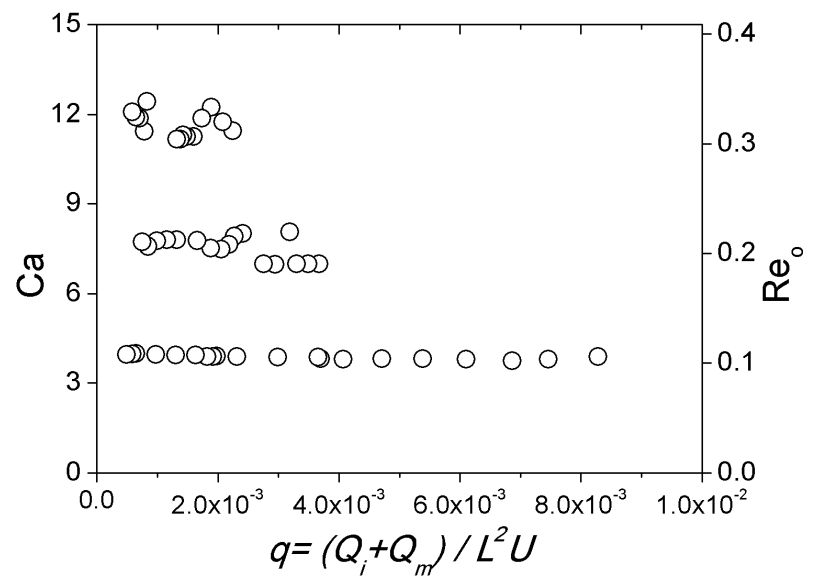

FIG. 3: The regime of periodic generation of micron-sized compound drops with diameters much smaller than the diameter of the injection tube is characterized by the conditions $R e_{o} \lesssim O(1), C a>C a^{*}$ and $q \ll 1$, see equations (2)-(4).

\section{CONDITIONS FOR THE PRODUCTION OF DOUBLE EMULSIONS}

Evangelio et al [1] have shown that double emulsions of newtonian liquids can be formed in a periodic way if the Reynolds number characterizing the flow of the continuous phase satisfy the condition,

$$
R e_{o}=\frac{\rho_{o} U L}{\mu_{o}} \lesssim O(1) .
$$

Moreover, the generation of stable long coaxial jets from which double emulsions are produced requires that

$$
C a=\frac{\mu_{o} U}{\sigma_{i}+\sigma_{m}}>C a^{*}\left(\lambda_{i}, q\right)
$$

where $\sigma_{i}$ is the interfacial tension coefficient between the inner and middle fluids, $\sigma_{m}$ represents the corresponding coefficient between the middle fluid and the continuous phase, $C a^{*}$ is the critical capillary number above which long steady jets are produced, $\lambda_{i}=\mu_{i} / \mu_{o}$ is the ratio of viscosities between the inner and outer fluids and $q$ denotes the flow rate ratio

$$
q=\frac{Q_{i}+Q_{m}}{Q_{o}}
$$




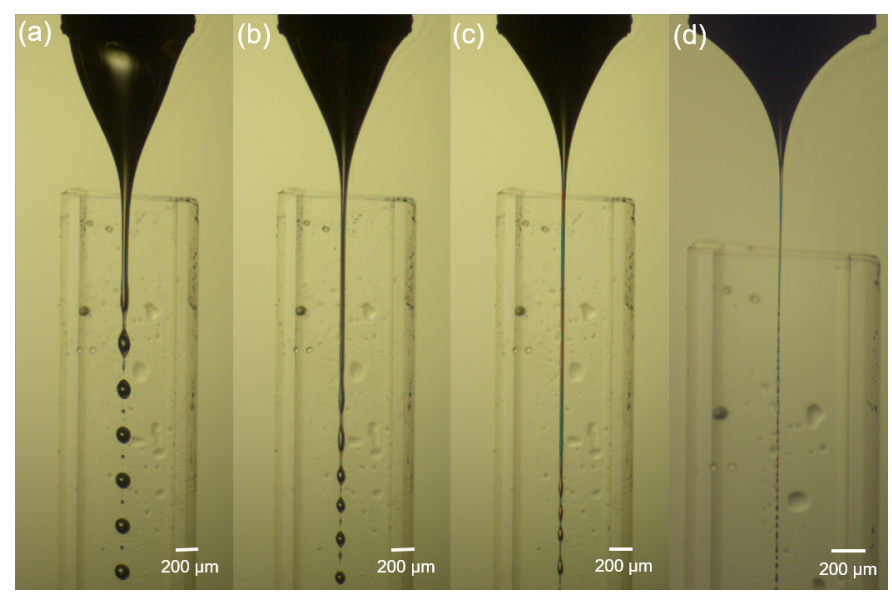

FIG. 4: The diameters of the produced shells, $D_{d}$, decrease upon increasing the velocity of the fluids at the extraction tube, $U . U$ is controlled through the pressure difference $P_{o}-P_{a}$, which depends linearly on $U$, see Fig. 2 .

The inner and middle flow rates, $Q_{i}$ and $Q_{m}$, are controlled using syringe pumps. (a-c): $Q_{i}=1 \mathrm{ml} / \mathrm{h}, Q_{m}=0.5$ $\mathrm{ml} / \mathrm{h}, \lambda_{i}=\mu_{i} / \mu_{o}=7.7 \times 10^{-4}$. (a) $U=0.035 \mathrm{~m} / \mathrm{s}, q=\left(Q_{i}+Q_{m}\right) /\left(U L^{2}\right)=0.012, R e_{o}=0.044, C a=1.35$. (b) $U$ $=0.08 \mathrm{~m} / \mathrm{s}, q=0.005, R e_{o}=0.1, C a=3.07$. (c) $U=0.15 \mathrm{~m} / \mathrm{s}, q=0.002, R e_{o}=0.19, C a=5.78$. (d) $Q_{i}=0.05$ $\mathrm{ml} / \mathrm{h}, Q_{m}=0.025 \mathrm{ml} / \mathrm{h}, \lambda_{i}=7.7 \times 10^{-4}, U=0.29 \mathrm{~m} / \mathrm{s}, q=2.8 \times 10^{-4}, R e_{o}=0.36, C a=11.15$.

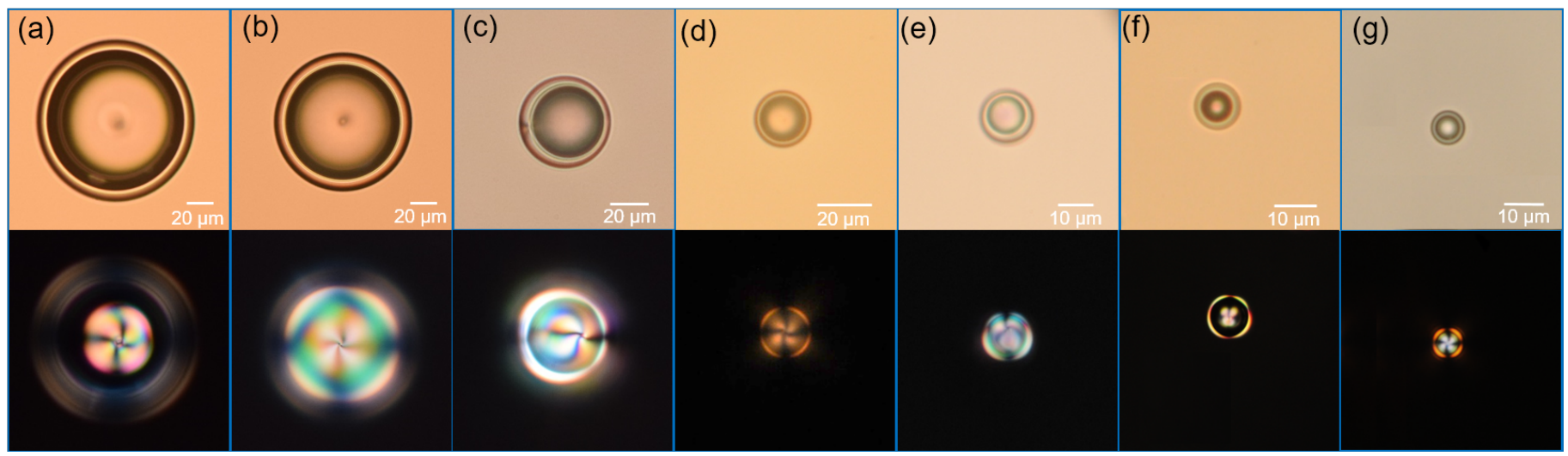

FIG. 5: Bright-field images (upper row) and cross-polarized images (lower row) of nematic shells produced with the CSW technique. The images show that the shell diameter, $D_{d}$, decreases with the flow-rate ratio $q$, defined in Eq. 4 . (a) $q=4.8 \times 10^{-3}, q_{i}=3.47 \times 10^{-3}, C a=3.07, R e_{o}=0.1, D_{d}=112 \mu \mathrm{m}, h=8 \mu \mathrm{m}$. (b) $q=1.8 \times 10^{-3}$, $q_{i}=9.26 \times 10^{-4}, C a=5.76, R e_{o}=0.19, D_{d}=96 \mu \mathrm{m}, h=7 \mu \mathrm{m}$. (c) $q=3.4 \times 10^{-4}, q_{i}=1.85 \times 10^{-4}, C a=5.76$, $R e_{o}=0.19, D_{d}=46 \mu \mathrm{m}, h=6 \mu \mathrm{m}$. (d) $q=2.4 \times 10^{-4}, q_{i}=4.79 \times 10^{-5}, C a=11.15, R e_{o}=0.36, D_{d}=20 \mu \mathrm{m}, h$ $=2 \mu \mathrm{m}$. (e) $q=1.25 \times 10^{-4}, q_{i}=3.47 \times 10^{-5}, C a=15.3, R e_{o}=0.504, D_{d}=14.5 \mu \mathrm{m}, h=2.5 \mu \mathrm{m}$. (f) $q=1.04 \times 10^{-4}, q_{i}=2.7 \times 10^{-5}, C a=15.3, R e_{o}=0.5, D_{d}=10.5 \mu \mathrm{m}, h=1.75 \mu \mathrm{m} .(\mathrm{g}) q=8.68 \times 10^{-5}$, $q_{i}=4.34 \times 10^{-5}, C a=12.3, R e_{o}=0.4, D_{d}=8.5 \mu \mathrm{m}, h=1.2 \mu \mathrm{m}$. Here, $h=0.5\left(D_{d}-D_{i}\right)$ indicates the thickness of the nematic shell and $q_{i}=Q_{i} /\left(U L^{2}\right)$.

with $Q_{o}=U L^{2}$. For values of the viscosity ratio $\lambda_{i} \sim O\left(10^{-3}\right)$ and of the flow rate ratio $q \ll 1$, it was theoretically found in [1] that double emulsions cannot be produced in a periodic and regular way if the value of the capillary number is below $C a^{*} \simeq 1$. This result was confirmed experimentally in [1], where it is demonstrated that, for values $C a<C a^{*}$, long liquid jets are not stable, a fact preventing the controlled and periodic production of micron-sized double emulsions.

To illustrate that tiny jets can be produced from the tip of a conical drop by hydrodynamic means [10, we decided not to add any type of surfactant to the liquids involved in the shell production process. In the absence of surfactants, stable double emulsions can still be produced if the following condition is fulfilled [20, 21]:

$$
\sigma_{i-o}>\sigma_{i}+\sigma_{m}
$$


where $\sigma_{i-o}$ is the value of the interfacial tension coefficient between the inner and outer liquids and $\sigma_{i}$ and $\sigma_{m}$ are defined after Eq. (3). Indeed, when condition (5) is satisfied, if a 'hole' appears in the shell, the interfacial tension force per unit length $\sigma_{i-o}$, which overcomes the sum of the interfacial tension forces $\sigma_{i}$ and $\sigma_{m}$, would tend to close this 'hole', preventing the inner droplet from being expelled outside. The use of the technique described above to
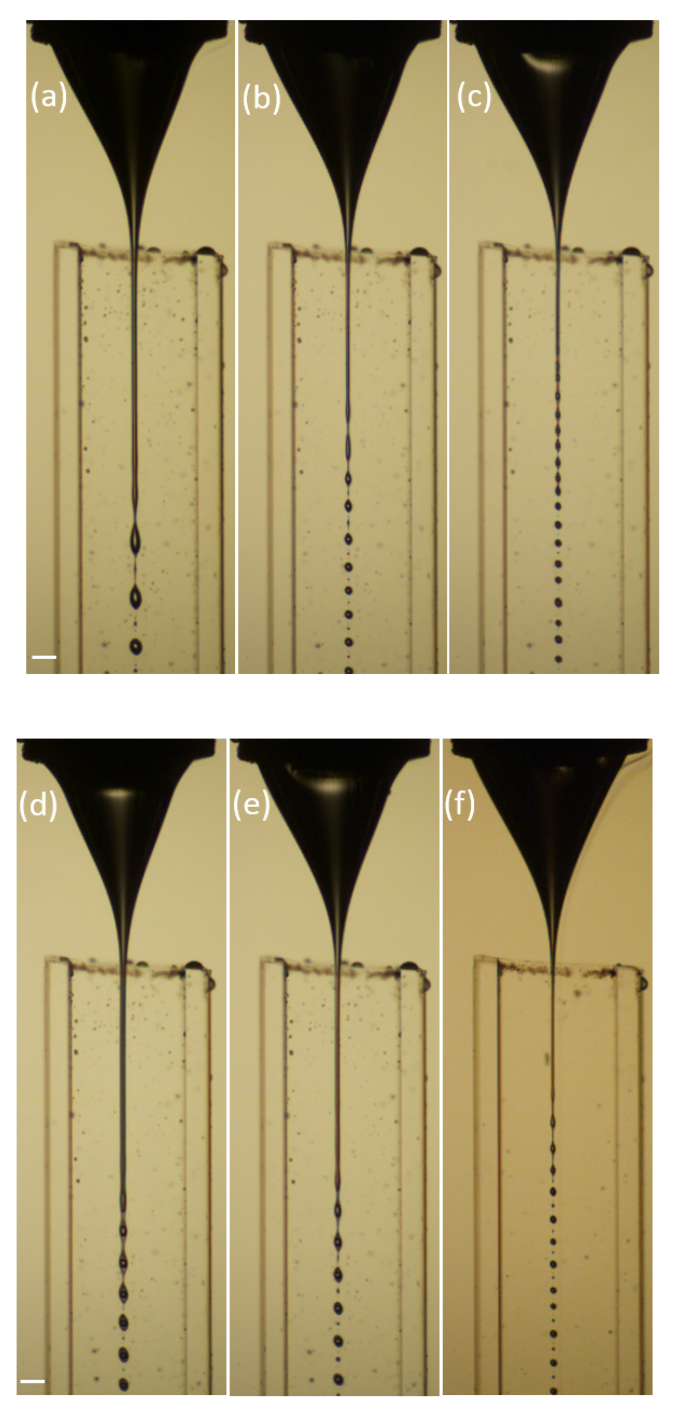

FIG. 6: The diameters of the produced shells, $D_{d}$, decrease when reducing either $Q_{i}$ or $Q_{m}$ while keeping the rest of parameters constant. (a-c) show the effect of varying $Q_{i}$ for fixed values of $U=0.08 \mathrm{~m} \mathrm{~s}^{-1}$, and $Q_{m} \simeq 0.25 \mathrm{ml} / \mathrm{h}$

( $C a=3.07, R e_{o}=0.1$ ): (a) $Q_{i}=1 \mathrm{ml} / \mathrm{h}$, (b) $Q_{i}=0.1 \mathrm{ml} / \mathrm{h}$ and (c) $Q_{i}=0.01 \mathrm{ml} / \mathrm{h}$. (d-f) show the effect of varying $Q_{m}$ for fixed values of $U=0.08 \mathrm{~m} \mathrm{~s}^{-1}$ and $Q_{i} \simeq 0.1 \mathrm{ml} / \mathrm{h},\left(C a=3.07, R e_{o}=0.1\right):\left(\right.$ d) $Q_{m}=0.9 \mathrm{ml} / \mathrm{h},(\mathrm{e})$ $Q_{m}=0.5 \mathrm{ml} / \mathrm{h}$ and (f) $Q_{m}=0.4 \mathrm{ml} / \mathrm{h}$. The scale bar indicates $200 \mu \mathrm{m}$.

produce liquid crystal shells requires to find a combination of liquids with physical properties such that the conditions expressed by Eq,2, Eq,3 and Eq,5 are concomitantly satisfied. We used 5CB (4-cyano-4'-pentylbiphenyl, Merck) as middle fluid because this liquid crystal has the advantage of having a nematic phase at room temperature and, in addition, its solubility with the outer fluid (glycerol) is quite small [22. The conditions expressed in Eq2 and Eq3 are simultaneously verified when pure glycerol (Sigma-Aldrich) is chosen as the continuous phase thanks to its large viscosity, $\mu_{o}$. Finally, the condition expressed by Eq 5 is satisfied when fluorinated oil (3M Novec) is used as the inner liquid because, with this choice, the values of the different interfacial tension coefficients, determined by means of the pendant drop method, are the following: $\sigma_{i} \simeq 7 \times 10^{-3} \mathrm{~N} \mathrm{~m}^{-1}, \sigma_{m} \simeq 19 \times 10^{-3} \mathrm{~N} \mathrm{~m}^{-1}$, and $\sigma_{i-o} \simeq 29 \times 10^{-3} \mathrm{~N} \mathrm{~m}^{-1}$. The values of density and viscosity for the different fluids are provided in Table 1 . 
TABLE I: Values of the material properties of the liquids used in the experiments as given by their manufacturers.

\begin{tabular}{lccc}
\hline \hline Fluid & Composition & $\rho\left(\mathrm{kg} \mathrm{m}^{-3}\right)$ & $\mu(\mathrm{cP})$ \\
\hline Inner & Fluorinated oil & 1614 & 0.77 \\
Middle & Liquid crystals (5CB) & 1008 & 30 \\
Outer & Glycerol & 1270 & 1000 \\
\hline \hline
\end{tabular}

\section{EXPERIMENTAL RESULTS}

The conical-shaped meniscus leading to the formation of shells is established for a range of values of $R_{o}, C a$ and $q$, respectively defined in Eqs 2, 4 . Fig 3 , which shows the values of $R e_{o}, C a$ and $q$ covered in our experimental study, reveals that double emulsions are indeed produced in a stable way for $R e_{o} \lesssim 1$ and $C a>1$, in accordance with Eqs 2 3 Due to the fact we are interested in exploring the small-size limit of the technique, we restricted our study to values of the flow rate ratio $q \ll 1$, being this the reason why there are no experimental points neither in the top-right part of Fig. 3 nor for larger values of $q$. In these regions, the diameters of the double emulsions produced would be larger than those reported here.

Fig. 4 shows how the shape of the meniscus evolves as function of $P_{o}-P_{a}$ for fixed values of $Q_{i}$ and $Q_{m}$. The imposed pressure difference $P_{o}-P_{a}$ fixes the mean velocity $U$ at the extraction tube (there is a linear relationship between the two parameters). Larger values of $U$ lead to the sharpening of the meniscus and to the thinning of the jet emerging from its tip. Since the shells emerge as a consequence of the growth of capillary instabilities, their outer diameters, $D_{d}$, are similar to the corresponding jet diameters. By making $U$ sufficiently large, we have been able to produce, in a robust and reproducible way, shells with values of $D_{d}$ below $10 \mu m$, see the last panel in Fig 5 corresponding to the smaller value of the flow rate ratio. Remarkably, let us anticipate here that we show in the last section how reducing the diameter of the injection needle and adding surfactants helps stabilizing the compound jet, a slight modification of the setup that enables getting values of $D_{d} \sim 5 \mu \mathrm{m}$. Fig 5 shows that $D_{d}$ can be additionally

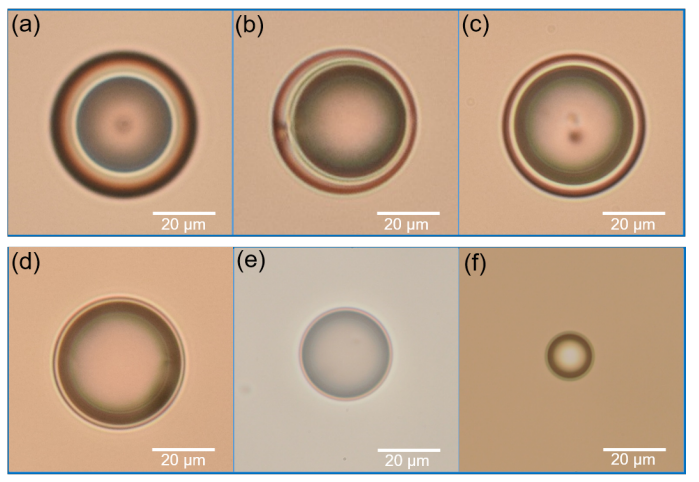

FIG. 7: (a-c): Bright field images of $5 \mathrm{CB}$ shells produced when $U=0.15 \mathrm{~ms}^{-1}$ is kept constant and $Q_{m}$ is varied. These images illustrate that the method employed here enables the production of double emulsions with practically the same outer diameter $D_{d}$ but different shell thicknesses: (a) $Q_{m}=0.12 \mathrm{ml} / \mathrm{h}, q=3.82 \times 10^{-4}, q_{i}=1.85 \times 10^{-4}$, $D_{d}=43 \mu \mathrm{m}, h=6.5 \mu \mathrm{m}$. (b) $Q_{m}=0.1 \mathrm{ml} / \mathrm{h} q=3.47 \times 10^{-4}, q_{i}=1.85 \times 10^{-4}, D_{d}=46 \mu \mathrm{m}, h=5.5 \mu \mathrm{m}$. (c) $Q_{m}=$ $0.08 \mathrm{ml} / \mathrm{h} q=3.33 \times 10^{-4}, q_{i}=1.85 \times 10^{-4}, D_{d}=46 \mu \mathrm{m}, h=4 \mu \mathrm{m}$. Figures (d)-(f) show that double emulsions with virtually the same thickness but different outer diameters can be produced using the CSW technique: (d) $Q_{i}=0.1$ $\mathrm{ml} / \mathrm{h} Q_{m}=0.07 \mathrm{ml} / \mathrm{h}, U=0.165 \mathrm{~ms}^{-1}, q=2.86 \times 10^{-4}, q_{i}=1.68 \times 10^{-4}, D_{d}=42 \mu \mathrm{m}, h=1.5 \mu \mathrm{m}$. (e) $Q_{i}=0.08$ $\mathrm{ml} / \mathrm{h} Q_{m}=0.03 \mathrm{ml} / \mathrm{h}, U=0.19 \mathrm{~ms}^{-1}, q=1.625 \times 10^{-4}, q_{i}=1.18 \times 10^{-4}, D_{d}=32 \mu \mathrm{m}, h=1.5 \mu \mathrm{m}$ (f) $Q_{i}=0.08$ $\mathrm{ml} / \mathrm{h} Q_{m}=0.08 \mathrm{ml} / \mathrm{h}, U=0.4 \mathrm{~ms}^{-1}, q=1.04 \times 10^{-4}, q_{i}=5.55 \times 10^{-5}, D_{d}=16 \mu \mathrm{m}, h=2 \mu \mathrm{m} . q_{i}=Q_{i} /\left(U L^{2}\right)$.

controlled by tuning $q$, defined in Eq. 4 , which depends on $Q_{o}(\mathrm{U}), Q_{m}$ and $Q_{i}$. Therefore, $D_{d}$ can be modified by changing $Q_{i}$ and $Q_{m}$ while keeping $U$ constant. Indeed, Fig. 6 shows that a decrease in $Q_{i}$ or $Q_{m}$ does not impact the shape of the meniscus much, but significantly reduces the jet diameter.

Moreover, Fig 7 reveals that, with the CSW technique, the outer diameter $D_{d}$ and the shell thickness can be controlled independently. Indeed, Figs. 7 (a)-(c) show that the thickness of the nematic shell can be reduced by decreasing $Q_{m}$, while Figs. 7(d)-(f) show that very thin shells, with a constant thickness of $\sim 1 \mu m$ but different outer 
diameters, can be produced for sufficiently small values of $q$ and $q_{i}$, with $q_{i}$ defined as

$$
q_{i}=\frac{Q_{i}}{U L^{2}} \text {. }
$$

Because of the high stability of the production regimes, all the samples show a small size dispersion which, as it can

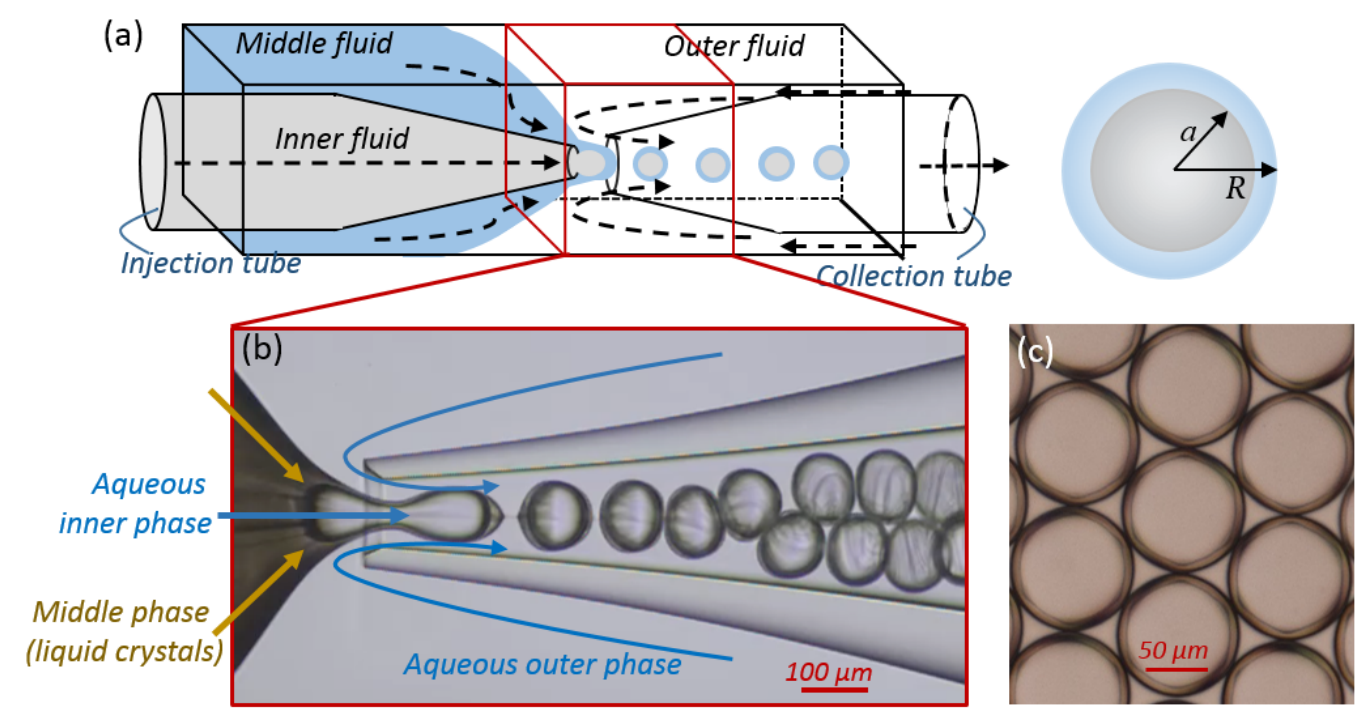

FIG. 8: (a) Left: schematic of a glass capillary device that combines co-flow and flow focusing. Right: schematic of a nematic liquid crystal shell, with an inner droplet of radius $a$ that is contained inside a bigger liquid crystalline droplet of radius $R$. (b) Production of liquid crystal shells in a glass capillary device. (c) Optical micrograph showing liquid crystal shells, with diameters of the order of the diameter of the constriction through which the three liquids flow.

be appreciated from the capillary breakup of the jets depicted in figures 4 and 6 is, at the outside, of the order of $10 \%$ the mean diameter of the droplets produced. Let us point out that the polydispersity index could be reduced if the jet was excited harmonically, but we have not explored this possibility here and the droplets generated in this study result from the growth of Rayleigh-Plateau capillary perturbations naturally induced by the ambient noise. Hence, the wavelength of the capillary wave leading to the disruption of the jet into drops is the one with the maximum growth rate, as we will show in the following section.

Now we discuss whether more standard methods, such those involving axi-symmetric glass capillary devices, would be able to reproduce our results. In order to show that our technique is unique in this aspect, let us consider a glass coaxial device like the one illustrated in Fig 8 , of the type described in [4, with a square capillary of width $L$ and a circular capillary of outer radius $L / 2$ inserted into the former. Since, in this case, the capillary number should also satisfy the condition in equation (3), the values of $U, Q_{i}$ and $Q_{m}$ in the glass capillary device should be the same as in the present study. In the geometry described in [4, the outer fluid should flow through the four narrow channels formed at the corners of the square capillary when the circular capillary is inserted inside. The cross-sectional area and the perimeter of each of these four narrow passages are given, respectively, by $A=(L / 2)^{2}(1-\pi / 4)$ and $P=L(1+\pi / 4)$ and hence, the hydraulic diameter of each of these four narrow constrictions, defined as the ratio $4 A / P$, is

$$
\bar{L}=L \frac{1-\pi / 4}{1+\pi / 4}
$$

Making use of equation (1), the relationship between the pressure drop and the outer flow rate is

$$
\begin{aligned}
& \frac{U L^{2}}{4} \simeq \frac{\pi \bar{L}^{4}}{128 \mu_{o}} \frac{P_{o}-P_{a}}{L_{T}} \rightarrow \frac{P_{o}-P_{a}}{L_{T}} \simeq \\
& \simeq \frac{128 \mu_{o}}{\pi L^{2}} U \times\left[\frac{1}{4}\left(\frac{1+\pi / 4}{1-\pi / 4}\right)^{4}\right],
\end{aligned}
$$


and hence, the pressure drop needed to make the outer fluid flow through the four narrow constrictions in the glass capillaries reported in [4] would be

$$
P_{o}-P_{a} \sim 5000 \frac{10 \sigma}{L} \frac{L_{T}}{L} \sim O\left(10^{7}\right) P a,
$$

where we have taken into account the fact that $\mu_{o} U \sim \sigma$ (see figure 3) and where we have used as a characteristic values: $L \sim 10^{-3} \mathrm{~m}, L_{T} / L \sim 10$ and $\sigma \sim 3 \times 10^{-2} \mathrm{~N} \mathrm{~m}^{-1}$. Clearly, the excessively large pressure drop given in equation (9), which is hundreds of times larger than the values of the pressure difference $P_{o}-P_{a}$ employed here, prevent reaching the high values of $U$ required to generate the long liquid jets depicted in Fig 4 . To the best of our knowledge, none of the numerous contributions in the literature that make use of hydrodynamic forcing to produce double emulsions 4, 5, 14 report the controlled production of double emulsions with diameters below $10 \mu \mathrm{m}$, and the reason for this is due to the extremely high values of the forcing pressure difference $P_{o}-P_{a}$ needed to produce a long jet. Hence, since the diameters of the compound droplets obtained using other techniques is fixed by geometrical dimensions, as Fig. 8 clearly illustrates [4, 5, the alternative would be to build devices with geometrical dimensions of just a few microns width, being the two main drawbacks of this hypothetical choice the large pressure drops needed to make the fluids flow through the tiny orifices and, also, that the resulting devices would be very prone to clogging. Let us point out here that the main advantages of using glass capillary or PDMS devices for producing double emulsions [4, 5, 14, are that the polydispersity index of the resulting compound drops is smaller and more controllable than in CSW and also that the resulting emulsion is much more concentrated.

Another important aspect of CSW is that the liquids flow through a long tube, not through an orifice, which further stabilizes the issued jet with respect to flow-focusing. Indeed, the use of a long tube ensures that the resulting compound jet highly stretches in the downstream direction, even within the long extraction capillary, a fact that strongly stabilizes the jet because it inhibits the growth of capillary perturbations, lowering the value of $C a^{*}$ [1]. In flow-focusing devices, however, a very short extraction orifice is used -see [23] and references therein for an in-depth discussion about the generation of emulsions using flow-focusing devices- a fact limiting the spatial extension of the region where the jet stretches downstream; consequently, the values of $C a^{*}$ in these types of devices are larger than those found in CSW (see Fig. 15 in [1]).

\section{ANALYSIS OF EXPERIMENTS}

This section is devoted to scale the drop production frequency $f$, the outer diameter $D_{d}$ and the shell thickness as a function of $U, Q_{i}$ and $Q_{m}$. The first step to express the shell production frequency, $f$, as a function of the control parameters, is to predict the dimensionless radius of the inner jet, $r_{\infty}=R_{\infty} / L$, with $R_{\infty}$ the corresponding dimensional value. Let us point out that the value of the dimensionless radius $r_{\infty}$ corresponds to the one in a region located far downstream the entrance of the extraction tube, where the coaxial jet is cylindrical and breaks into droplets (see Fig. 4). The use of the continuity equation for the inner fluid yields [1,

$$
\begin{aligned}
& q_{i}=\frac{Q_{i}}{U L^{2}}=\left[2-8 r_{\infty}^{2}\right] \pi r_{\infty}^{2}+4 \frac{\pi r_{\infty}^{4}}{\lambda_{i}} \simeq 2 \pi r_{\infty}^{2}+4 \frac{\pi r_{\infty}^{4}}{\lambda_{i}} \\
& \Rightarrow r_{\infty}=\left[\frac{\lambda_{i}}{4}\left(-1+\sqrt{1+\frac{4 q_{i}}{\pi \lambda_{i}}}\right)\right]^{1 / 2} \quad \text { with } \quad \lambda_{i}=\mu_{i} / \mu_{o} \ll 1
\end{aligned}
$$

where it has been taken into account that $Q_{i}$ results from the addition of the flow rate corresponding to a plug flow of velocity $2 U$ plus the flow rate corresponding to the parabolic Poiseuille flow. The Poiseuille flow is created by the pressure gradient that exists within the extraction tube. Once the dimensionless radius of the innermost jet, $r_{\infty}$, is expressed as a function of the control parameters through Eq 10, we now turn to determine the value of the capillary wavelength, $\ell_{0}$, leading to the formation of a droplet of diameter $D_{i}$. For that purpose, we make use of the idea that the innermost part of the coaxial jet breaks into drops due to the growth of a Rayleigh-Plateau capillary instability, and this process triggers the capillary break-up of the middle liquid, leading to the formation of micron-sized shells.

The capillary wavelength is thus calculated as

$$
\ell_{0}=\frac{2 \pi L}{k_{\max }} r_{\infty}
$$


with $k_{\max }$ the dimensionless wavenumber that maximizes the temporal growth rates of capillary perturbations i.e., Real $(-i \omega)$, with $\omega(k)$ given by the dispersion relation

$$
\begin{aligned}
D(\omega, k) & =-i \omega\left(2+\frac{k^{2}}{4 \lambda_{i}}\right) \\
& +i k\left[2 u_{x \infty}+f_{\infty} r_{\infty}^{2}\left(4-\frac{2}{\lambda_{i}}\right)\right]-\frac{k^{2}}{8 \lambda_{i} C a} \\
& +i k^{3}\left(\frac{u_{x \infty}}{4 \lambda_{i}}+\frac{f_{\infty} r_{\infty}^{2}}{4 \lambda_{i}}\right)+\frac{k^{4}}{8 \lambda_{i} C a}=0,
\end{aligned}
$$

deduced in 1 using slender-body theory. In spite of its simplicity, Eq. 12 reproduces, in the limit $\lambda_{i} \ll 1$, the results obtained using the well known but much more involved dispersion relation due to Tomotika [24. In Eq, 12, $k$ is a real number and denotes the dimensionless wavenumber, $\omega$ is the dimensionless complex frequency $u_{x \infty}=2, f_{\infty}=-8$ and $r_{\infty}$ is the solution of the continuity equation 10 Also, note that the capillary number in Eq 12 , defined in Eq3. expresses that the inner jet breaks as a consequence of the growth of capillary disturbances with an effective interfacial tension coefficient resulting from the addition of the inner and intermediate interfacial tension coefficients [25. Therefore, using Eq $12, k_{\max }$ is given by

$$
\begin{aligned}
\operatorname{Max}[\operatorname{Real}(-i \omega)] & =\operatorname{Max}\left(\frac{1}{8 \lambda_{i} C a} \frac{k^{2}-k^{4}}{2+k^{2} /\left(4 \lambda_{i}\right)}\right) \\
& =\operatorname{Max}\left(\frac{k^{2}-k^{4}}{8 \lambda_{i}+k^{2}}\right) \\
& \Rightarrow k_{\max }=\left[-8 \lambda_{i}+8 \lambda_{i} \sqrt{1+\left(8 \lambda_{i}\right)^{-1}}\right]^{1 / 2} \\
& \Rightarrow k_{\max } \simeq\left(8 \lambda_{i}\right)^{1 / 4} \text { for } \quad \lambda_{i} \ll 1 .
\end{aligned}
$$

The drop production frequency is thus given by

$$
f=\frac{2 U}{\ell_{0}}=\frac{U k_{\max }}{\pi L r_{\infty}} \simeq \frac{\left(8 \lambda_{i}\right)^{1 / 4}}{\pi} \frac{U}{L r_{\infty}}=K_{1} \frac{U}{L r_{\infty}},
$$

with $K_{1} \simeq 0.09$ for $\lambda_{i}=7.7 \times 10^{-4}$ and where we have made use of Eqs 11 and 13 .

To test this relationship in our system, we plot the shell production frequency $f$ as a function of $U /\left(L r_{\infty}\right)$ at different production regimes, see Fig. 9(a). Here, $r_{\infty}$ is calculated from Eq. 10 with $q_{i}$ given by Eq. (6), and $f$ is measured as the number of shells produced at a given time interval, $n_{\text {shell }} / N \Delta t$, where $N$ is the number of video frames in the considered time interval and $1 /(\Delta t)$ is the camera frame rate. We obtain a linear relationship with a proportionality constant equal to $K_{1}=0.089 \pm 0.003$, the expected value from Eq. 14, validating our approach.

Once the drop formation frequency is known, and in order to predict the inner, $D_{i}$, and outer, $D_{d}$, diameters of the drops bounding the shell, continuity demands that

$$
\begin{aligned}
& \frac{\pi D_{i}^{3}}{6} f=Q_{i} \Rightarrow K_{1} \frac{\pi D_{i}^{3}}{6} \frac{U}{L r_{\infty}}=Q_{i} \\
& \Rightarrow \frac{D_{i}}{L}=\left(\frac{6 r_{\infty}}{\pi K_{1}}\right)^{1 / 3} q_{i}^{1 / 3}, \\
& \frac{\pi D_{d}^{3}}{6} f=Q_{i}+Q_{m} \Rightarrow K_{1} \frac{\pi D_{d}^{3}}{6} \frac{U}{L r_{\infty}}=Q_{i}+Q_{m} \\
& \Rightarrow \frac{D_{d}}{L}=\left(\frac{6 r_{\infty}}{\pi K_{1}}\right)^{1 / 3} q^{1 / 3},
\end{aligned}
$$

with $f$ given by Eq 14 and $q$ and $q_{i}$ defined, respectively, in Eqs. 4 and 6 .

The experimentally measured values of $D_{i}$ and $D_{d}$ are compared with the predictions given by Eq. 15 in Fig 9 (b) and Fig 9 (c), respectively. We find an excellent agreement between experiments and theory, which demonstrates that the CSW enables the production of double emulsions in a highly controlled way.

In the experiments described above, the inner and middle liquids were injected into the pressurized chamber using syringe pumps. In this way, we have been able to produce liquid crystal shells with $D_{d} \gtrsim 10 \mu \mathrm{m}$. To further decrease 

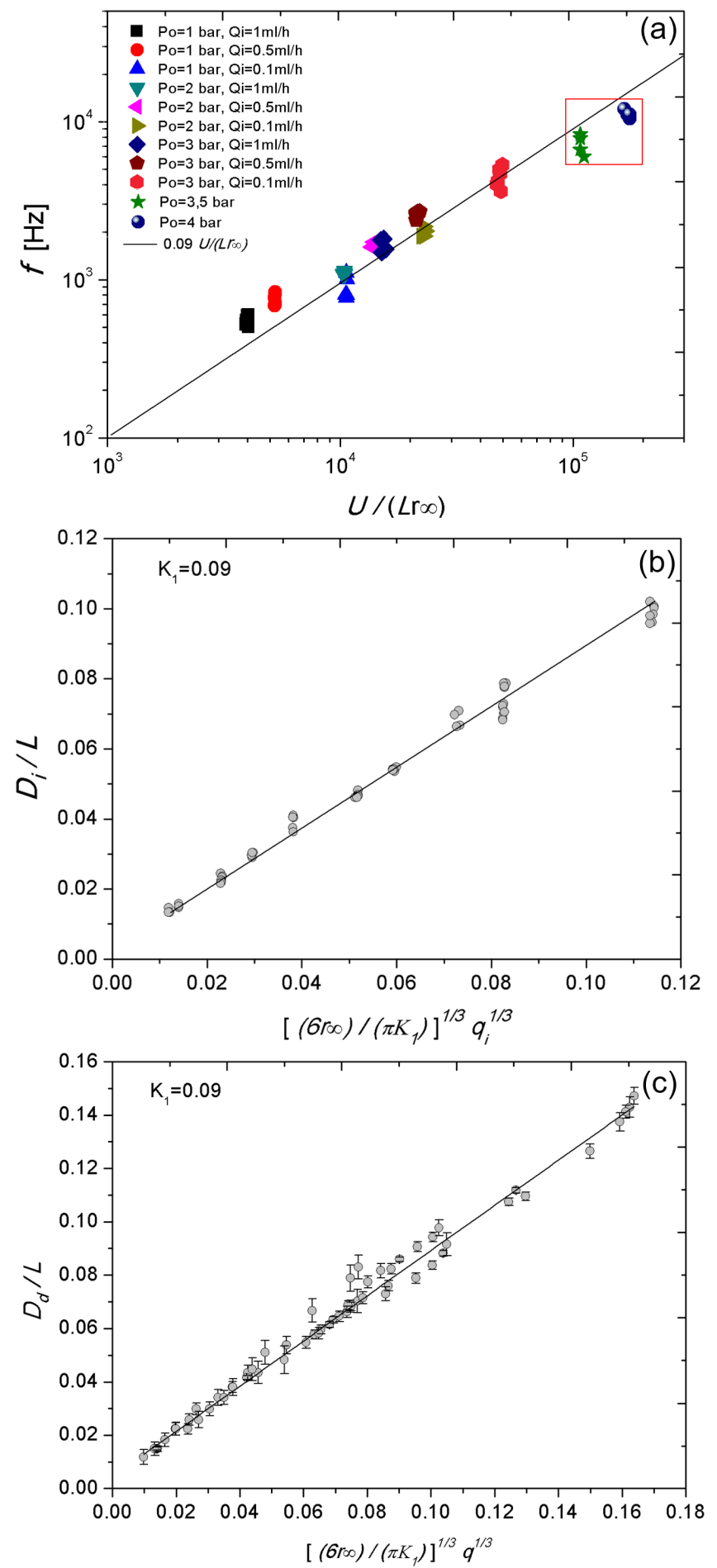

FIG. 9: (a) The experimentally measured frequencies depend linearly on $U /\left(L r_{\infty}\right)$, as predicted by equation (14). (b)-(c) The experimentally measured inner and outer diameters of the double emulsion, $D_{i}$ and $D_{d}$ respectively, are compared with the predictions given by equation 15, finding an excellent agreement between observations and theory. The red boxes indicate those experiments for which the inner and intermediate flow rates have not been set using syringe pumps, but varying the pressure differences $P_{i}-P_{o}$ and $P_{m}-P_{o}$, which depend linearly on $Q_{i}$ and $Q_{m}$, respectively. 
$D_{d}$, the flow rates of both the inner and middle fluids need to be extremely small (below $0.02 \mathrm{ml} / \mathrm{h}$ ). But, if these tiny flow rates were imposed using syringe pumps, the perturbations introduced by the motors would destabilize the coaxial jet before the double emulsion is generated. To circumvent this issue, we can reduce the noise introduced into the system by imposing pressure differences to the inner and middle fluids instead. Indeed, tuning manually the values of $P_{i}-P_{o}$ and $P_{m}-P_{o}$ with the pressure regulators allows us to indirectly control the values of $Q_{i}$ and $Q_{m}$, see Fig. 1. Indeed, because of the long injection tubes used and the low flow rates imposed, there is a linear relationship of the type expressed by Eq. 1 between the imposed pressure differences and the corresponding flow rates, $Q_{i}$ and $Q_{m}$. By controlling $Q_{i}$ and $Q_{m}$ through $P_{i}-P_{o}$ and $P_{m}-P_{o}$, we get both appreciably larger production frequencies and clearly smaller shell diameters, as highlighted with a red box in Fig. 9. These new points also follow the scaling laws established in Eq 14 and $\mathrm{Eq} 15$

\section{ANALYSIS OF THE TOPOLOGICAL DEFECTS OF THE NEMATIC SHELLS PRODUCED}

One main goal of modern material science is to produce complex three-dimensional architectures from the selfassembly of pre-designed colloidal building blocks. This would enable producing materials whose optical index is modulated at a meso-scale. By designing the proper building blocks, we might be able to generate macroscopic colloidal architectures with extraordinary optical properties, such as metamaterials or photonic crystals [26. Classical colloids interact isotropically and usually lead to closed-packed crystalline structures [27, 28]. More interesting symmetries can emerge if the building blocks are able to interact in an anisotropic way.
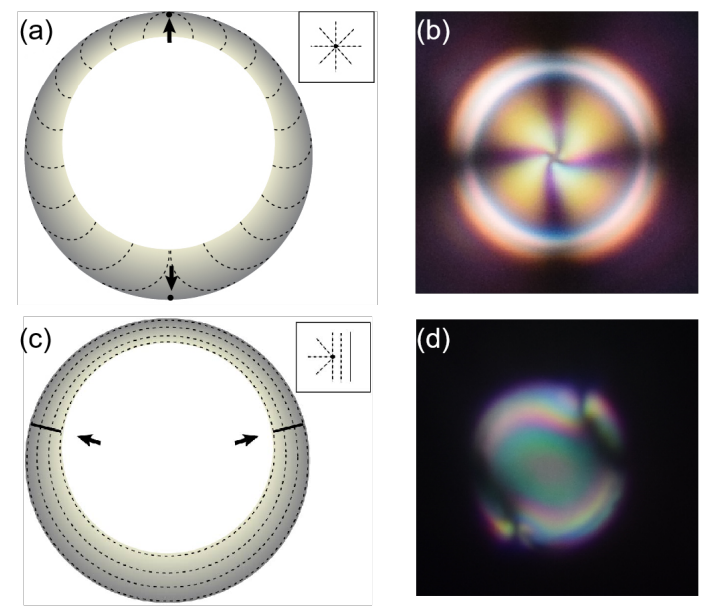

FIG. 10: Schematic representation of a shell with (a) hybrid boundary conditions and (c) planar boundary conditions. The dashed lines represent the director field across the shell. The arrows point out the presence of topological defects. In (a), the topological defects are +1 surface defects or boojums, inducing a $2 \pi$-rotation of the director, as represented in the inset, which shows a top view of the defect. In (c), the defects are $+1 / 2$ disclination

lines, inducing a $\pi$-rotation of the director. Note that the other two $+1 / 2$ disclination lines needed to fullfil the topological constrains in (c) are in a different plane, and thus, not represented. (b) and (d) are cross-polarized images showing the top view of two shells with hybrid and planar boundary conditions respectively. Fluorinated oil is encapsulated in (b) whereas water with 1\% wt of polyvinyl alcohol (PVA) is encapsulated in (d). Figures (b) and (d) are included just to illustrate the two types of boundary conditions that can be found depending on whether fluorinated oil or water+PVA is encapsulated, being this the reason why the exact dimensions of each compound droplet is irrelevant and, hence, not indicated.

The defect structure in the shell strongly depends on the orientation of the liquid crystal molecules at the shell boundaries. In our experiments, the shells have been produced using fluorinated oil and glycerol as inner and outer phases of the double emulsion, respectively. Fluorinated oil induces normal (homeotropic) anchoring of the liquid crystal molecules at the interface, while glycerol imposes planar anchoring, thus leading to a global hybrid anchoring. These boundary conditions result in the molecular organization reported in 29] and shown in Fig. 10(a), where the director field is represented by dashed lines across the shell. The planar anchoring induces the formation of two pointdefects, pointed out by arrows in Fig. 10(a), on the outer sphere at one diameter distance. These defects have +1 winding number or topological charge, indicating the $2 \pi$-rotation induced by the defect in the director $n$, see the inset 
in Fig. 10(a). The total topological charge on the outer sphere is +2 , consistently with the topological constraints [30. The homeotropic anchoring on the outer surface makes the director tilt upwardly from the inner sphere towards the outer one, where the presence of defects is not required. Fig. 10(b) shows a top view of an experimental shell with hybrid boundary conditions under cross-polarizers. The crossing of the four black brushes indicates the presence of $\mathrm{a}+1$ defect at the top of the outer droplet (the other one is placed at the bottom). This configuration seem to be present in the whole range of sizes studied, see Fig 5 (lower row).

Imposing planar boundary conditions on both interfaces of the shell results in the formation of a larger number of possible defect configurations [6] interesting for applications. We demonstrate that the CSW technique is suitable to produce shells with planar boundary conditions by replacing the fluorinated oil by a solution of water with 1\% wt of polyvinyl alcohol (PVA), which is well known to produce planar anchoring. This combination of fluids leads to more unstable jets than those produced when fluorinated oil is used and surfactants are avoided. To overcome this problem, we used a new coaxial needle (purchased from Ramé-Hart Instrument) with smaller dimensions (inner diameter $178 \mu m$, and outer diameter $406 \mu \mathrm{m})$. Reducing the size of the injection needle allowed us to stabilize the jet and reduce the diameters of the shells even further. With this new geometry and these new liquids, we were able to reach values of the radius of curvature close to $2 \mu \mathrm{m}$, as shown in Figure 11. Yet, we could not generate smaller shells as a consequence of the small asymmetries existing between the two concentric injection tubes, a purely geometrical issue which we could not circumvent in this study, but will be hopefully solved using other fabrication tecniques, like 3D printing.

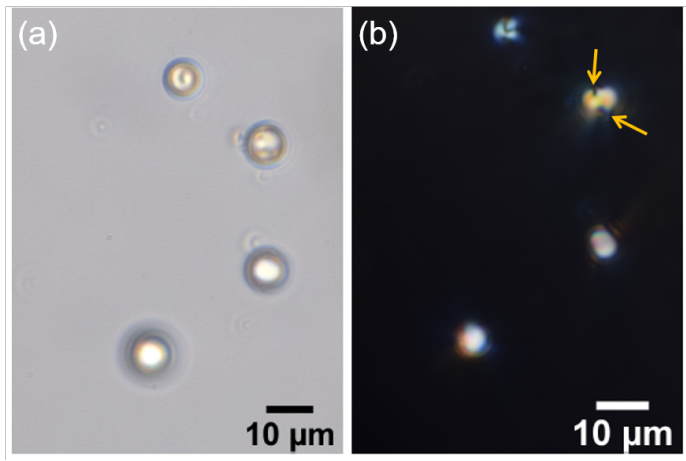

FIG. 11: Top view of shells with planar boundary conditions and diameters below 10 microns: (a) bright field and

(b) cross-polarization. The two black brushes (indicated by yellow arrows) emerging from the defects in cross polarization seem to indicate a $+1 / 2$ topological charge. The smallness of the shells produced and the visualization technique employed prevents us from asserting, with no kind of doubt, that the defects correspond to $+1 / 2$ disclination lines.

The shells produced using PVA were very stable, even when collected in the simplest possible way, i.e., placing a glass slide right underneath the extraction tube. Because of their planar boundary conditions, the molecular organization inside these shells is expected to have an essentially two-dimensional structure, as it is suggested by the images shown in Fig. 10(c)-(d). It has been shown that the type of defect structure in shells with planar boundary conditions depends on the thickness of the shell [7]. The question that remains open is how the shell size influences the defect structure. We observe that in our smallest shells (which are also thinner), the defects observed seem to correspond to $+1 / 2$ disclination lines spanning the shell, see Fig. 11. Two of these lines are represented in Fig. 10(c) (see arrows for a side view and inset for a top view). However, the resolution of the optical methods typically employed to characterize the shells and the smallness of the droplets produced did not allow us to undoubtitatively conclude that the defects correspond to $+1 / 2$ disclination lines. A quantitative study about the effect of size and curvature on the shell defect structure is desirable, but a characterization with higher resolution techniques would be necessary, which is outside the scope of the present contribution.

\section{CONCLUSIONS}

We have made use of the Confined Selective Withdrawal (CSW) technique, based on the capillary breakup of highly stretched long coaxial capillary jets, to produce double emulsions with the smallest diameters reported so far and at frequencies exceeding $10 \mathrm{KHz}$. In CSW, the diameters of the double emulsions are not set by the sizes of the injectors, 
in contrast with more standard microfluidic techniques based on axi-symmetric glass capillary devices [4] or PDMS chips [5]. In this way, we avoid using tiny injection orifices or channels, which are prone to clogging and limit the size of the emulsions that can be fabricated. In addition, the relatively low values of the forcing pressures needed to make the three immiscible liquids flow through the device, favors the production of double emulsions with outer diameters below $10 \mu \mathrm{m}$ and with shell thicknesses that can be reduced down to $1 \mu \mathrm{m}$. We have shown that the slender-body theoretical predictions for the outer diameters of the shells, their thicknesses and the generation frequencies are in fair agreement with the experimental observations. In summary, this work provides a robust method to fabricate, in a controlled and continuous way, the smallest micron-sized shells reported up to date.

\section{ACKNOWLEDGEMENTS}

This work has been funded by the French National Research Agency (Grant 13-JS08-0006-01), by the Spanish MINECO under projects DPI2014-59292-C3-2-P and DPI2017-88201-C3-1-R, partly financed by European funds, and by the Chinese Scholarship Council. The authors wish to acknowledge Prof. I.G. Loscertales for providing us with the coaxial injectors.

* jgordill@us.es

[1] A Evangelio, F Campo-Cortés, and J. M. Gordillo, "Simple and double microemulsions via the capillary breakup of highly stretched liquid jets," Journal of Fluid Mechanics, 804, 550-577 (2016).

[2] I. G. Loscertales, A. Barrero, I Guerrero, R Cortijo, M Marquez, and AM Ganan-Calvo, "Micro/nano encapsulation via electrified coaxial liquid jets," Science, 295, 1695-1698 (2002).

[3] A. Barrero and I. G. Loscertales, "Micro-and nanoparticles via capillary flows," Annu. Rev. Fluid Mech., 39, 89-106 (2007).

[4] AS Utada, EL Lorenceau, DR Link, PD Kaplan, HA Stone, and DA Weitz, "Monodisperse double emulsions generated from a microcapillary device," Science, 308, 537-541 (2005).

[5] M.L. Eggersdorfer, W. Zheng, S Nawar, C Mercandetti, A Ofner, I Leibacher, S Koehler, and D.A. Weitz, "Tandem emulsification for high-throughput production of double emulsions," Lab on a Chip, 17, 936-942 (2017).

[6] A. Fernández-Nieves, V. Vitelli, A.S. Utada, D. R. Link, M. Márquez, D. R. Nelson, and D. A. Weitz, "Novel defect structures in nematic liquid crystal shells," Physical review letters, 99, 157801 (2007).

[7] T. Lopez-Leon, V. Koning, KBS Devaiah, V. Vitelli, and A Fernandez-Nieves, "Frustrated nematic order in spherical geometries," Nature Physics, 7, 391-394 (2011).

[8] Teresa Lopez-Leon and Alberto Fernandez-Nieves, "Drops and shells of liquid crystal," Colloid and Polymer Science, 289, $345(2011)$.

[9] J. P. F. Lagerwall and G. Scalia, "A new era for liquid crystal research: Applications of liquid crystals in soft matter nano-, bio- and microtechnology," Curr. Appl. Phys., 12, 1387 (2012).

[10] R. Suryo and O. A. Basaran, "Tip streaming from a liquid drop forming from a tube in a co-flowing outer fluid," Physics of Fluids, 18, 082102 (2006).

[11] AG Marin, F Campo-Cortés, and J. M. Gordillo, "Generation of micron-sized drops and bubbles through viscous coflows," Colloids and Surfaces A: Physicochemical and Engineering Aspects, 344, 2-7 (2009).

[12] A. Evangelio, F. Campo-Cortes, and J. M. Gordillo, "Pressure gradient induced generation of microbubbles," Journal of Fluid Mechanics, 778, 653-668 (2015).

[13] I. G. Loscertales, A. Barrero, M. Márquez, R. Spretz, R. Velarde-Ortiz, and G. Larsen, "Electrically forced coaxial nanojets for one-step hollow nanofiber design," Journal of the American Chemical Society, 126, 5376-5377 (2004).

[14] S.A. Nabavi, G.T Vladisavljevic, Gu S., and E.E. Ekanem, "Double emulsion production in glass capillary microfluidic device: Parametric investigation of droplet generation behaviour," Chemical Engineering Science, 130, 183 - 196 (2015).

[15] D. R. Nelson, "Toward a tetravalent chemistry of colloids," Nano Letters, 2, 1125-1129 (2002).

[16] TC Lubensky and J. Prost, "Orientational order and vesicle shape," Journal de Physique II, 2, 371-382 (1992).

[17] Gretchen A. DeVries, Markus Brunnbauer, Ying Hu, Alicia M. Jackson, Brenda Long, Brian T. Neltner, Oktay Uzun, Benjamin H. Wunsch, and Francesco Stellacci, "Divalent metal nanoparticles," Science, 315, 358 (2007).

[18] V. Vitelli and D. R. Nelson, "Nematic textures in spherical shells," Phys. Rev. E, 74, 021711 (2006).

[19] J. D. Sherwood, "Tip streaming from slender drops in a nonlinear extensional flow," Journal of Fluid Mechanics, 144, 281-295 (1984)

[20] D. Bonn, J. Eggers, J. Indekeu, J. Meunier, and E. Rolley, "Wetting and spreading," Rev. Mod. Phys., 81, 739-805 (2009).

[21] L. D. Zarzar, V. Sresht, E. M Sletten, J. A. Kalow, D. Blankschtein, and T.M. Swager, "Dynamically reconfigurable complex emulsions via tunable interfacial tensions," Nature, 518, 520-524 (2015).

[22] I. I. Smalyukh, S. Chernyshuk, B. I. Lev, A. B. Nych, U. Ognysta, V. G. Nazarenko, and O. D. Lavrentovich, "Ordered droplet structures at the liquid crystal surface and elastic-capillary colloidal interactions," Phys. Rev. Lett., 93, 117801 (2004). 
[23] S.L. Anna, "Droplets and bubbles in microfluidic devices," Annual Review of Fluid Mechanics, 48, 285-309 (2016).

[24] S. Tomotika, "On the instability of a cylindrical thread of a viscous liquid surrounded by another viscous fluid," Proceedings of the Royal Society of London. Series A, Mathematical and Physical Sciences, 150, 322-337 (1935).

[25] A Chauhan, C Maldarelli, DT Papageorgiou, and DS Rumschitzki, "Temporal instability of compound threads and jets," Journal of Fluid Mechanics, 420, 1-25 (2000).

[26] O. D. Lavrentovich, "Liquid crystals, photonic crystals, metamaterials, and transformation optics," Proc. Natl. Acad. Sci., 108, 5143 (2011).

[27] V. N Manoharan, M. T Elsesser, and D. J. Pine, "Dense packing and symmetry in small clusters of microspheres," Science, 301, 483-487 (2003).

[28] F. Li, D. P Josephson, and A. Stein, "Colloidal assembly: the road from particles to colloidal molecules and crystals," Angewandte Chemie International Edition, 50, 360-388 (2011).

[29] T Lopez-Leon and A Fernandez-Nieves, "Topological transformations in bipolar shells of nematic liquid crystals," Physical Review E, 79, 021707 (2009).

[30] H. Poincare, "Sur les courbes dfinies par les equations diffrentielles," J. Math. Pures Appl., 1, $167-244$ (1885). 